Okoloba, M. M. ${ }^{1}$, Ogueji, I. A. ${ }^{2 *}$, Darroch, S. J. ${ }^{3}$, Ogueji, A. M. ${ }^{4}$

\title{
A Multinational Pilot Study on the Lived Experiences and Mental Health Impacts from the COVID-19 Pandemic
}

\author{
'The University of Buckingham, Department of Psychology, Yeomanry House, Buckingham, Buckinghamshire, MK18 1EG; and Insightful \\ Findings, United Kingdom \\ 2Department of Psychology, University of Ibadan, Ibadan, Nigeria; and Insightful Findings, United Kingdom \\ ${ }^{3}$ School of Psychology, University of Kent, Canterbury, Kent, CT2 7NP, United Kingdom \\ «Department of Sociology, University of Ibadan, Ibadan, Nigeria; and Insightful Findings, United Kingdom \\ *email: Ifeanyiogueji21agmail.com or researchteamragmail.com
}

DOI: 10.2478/gp-2020-0015

Received: 9 May 2020; Accepted: 30 May 2020

\begin{abstract}
Objectives: To conduct a pilot study on the lived experiences of people affected by the COVID-19 pandemic, and the mental health impacts of the COVID-19 pandemic on affected people.

Methods: This mixed methods study used random and snowball techniques to recruit 91 respondents (age range 18-58 years) from 14 countries. Accessing patients with COVID-19 was restricted because of the nature of the disease and the treatment they were receiving which limited their access to being selected for our pilot study. Therefore, our respondents were members of the general population who were directly or indirectly affected by the pandemic. Respondents completed online surveys consisting of the Generalised Anxiety Disorder-7 (GAD-7), Patient Health Questionnaire 9 (PHQ-9) and open-ended questions. Collected data were subjected to IBM SPSS statistics software (v. 22.0) and thematic analysis.

Results: Both quantitative and qualitative instruments reported very high feasibility, which is suggestive of an excellent feasibility of our mixed methods design. Second, we identified financial and time resources as major barriers to the recruitment process, and how to avert these in the main study was discussed. Third, COVID-19 pandemic had more anxious than depressive impacts on our respondents. We also found that more than half $(58,63.7 \%)$ of the total respondents showed high knowledge level of COVID-19, and the major sources from which they gained knowledge were television, radio, and online academic papers. Our qualitative insight showed five themes from the lived experiences of respondents from the COVID-19 pandemic. These themes were; changes in lifestyles, disruption of schooling activities, impact on options for health services, disruption of works and economy, and anxious and worrisome impacts, in that order.

Conclusion: Main study is feasible with minor addition required on our protocol. Given other findings, we recommend the need for adaptive coping skill training, and appropriate information sharing during pandemics. There is also the need for psychosocial preparation due to the uncertainty of pandemics. In addition, averting the likely adverse economic impacts from pandemics should be of concern to governments and other stakeholders during pandemics. Finally, we recommend that governments and other stakeholders should be cautious about the likelihood of limited health service options for the general public during pandemics.
\end{abstract}

\section{Keywords}

COVID-19 pandemic, Pilot study, Mixed methods design

\section{INTRODUCTION}

Coronavirus, which is the causal agent of the current deadliest disease, belongs to the family of viruses. Coronavirus is zoonotic, meaning it is transmitted between animals and humans (Adhikari et al. 2020). In 2019, a new report of coronavirus disease known as COVID-19 emerged in humans. It was believed to have started in Wuhan, China. The World Health Organization (WHO) declared it a public health emergency on
30 January, 2020. Further, the name COVID-19 was assigned to the coronavirus disease on 11 February, 2020, and it was declared a pandemic on 11 March, 2020 (WHO, 2020).

Some common symptoms recorded of COVID-19 are fever at $38^{\circ} \mathrm{C}$ or above, dry cough and difficulty breathing (Adhikari et al. 2020). The same report shows that patients may report a general feeling of being unwell, nasal congestion, sore throat, aches and pains. Some people also become infected without 
developing symptoms (asymptomatic). People infected with COVID-19 may recover, especially if they are a younger population or living without underlying illness (Rothan \& Byrareddy, 2020). This is suggestive that the aged population or persons living with underlying illness have poor chances of recovering from a COVID-19 infection. COVID-19, which is now a pandemic, has recorded over 3 million confirmed cases spread across 212 countries and 238,084 deaths worldwide at present and these figures are currently rising (WHO, 2020).

An implication of these figures that spread across 212 countries is suggestive that there likely would be varied lived experiences and health impacts arising from the novel COVID-19 pandemic among people who are directly or indirectly affected by the pandemic. In terms of the health impacts from pandemics, researchers have often argued that the mental health should often be a paramount focus for researchers interested in exploring the health impacts from pandemics (Nickell, et al. 2004; Cava, et al. 2005; Gardener \& Moallef, 2015; Park, et al. 2018; Xiang, et al. 2020). Further, the same researchers opined that, sufficient research into the experiences of everyone affected, and mental health impacts of pandemics on everyone affected, is underscored for informing appropriate intervention or protection programmes for everyone affected during pandemics.

Therefore, we intend to embark on a multinational study that explores the lived experiences of everyone affected by the novel COVID-19 pandemic, and the mental health impacts of the pandemic among everyone affected. Our intention is driven by the earlier cited literature which suggested this as the procedure for arriving at a sufficiently educated decision that is designed to offer intervention or protection programs for everyone affected during pandemics. Before embarking on our multinational study, it is worthy to acknowledge that scholars have strongly opined that a pilot study is imperative for gaining insight into what is actually required for our main multinational study that is aimed at informing appropriate intervention or protection programs for everyone affected by the novel COVID-19 pandemic (Maunder, 2009; Bidarbakhtnia, 2020). Further, some other scholars have also submitted that pilot studies offer increased likelihood of success for a main study by establishing its feasibility (Teijlingen \& Hundley, 2001; Thabane, et al. 2019; Malmqvist, et al. 2019). Thus, we decided to have this pilot phase as a starting point.

\section{SIGNIFICANCE OF STUDY}

With regard to the focus of our current study, there have been vast amounts of literature dedicated to the lived experiences of people during infectious disease outbreaks, and the mental health impacts of infectious disease outbreaks, (Lazarus, et al. 2002; Sprang \& Silman, 2013; Gardener \& Moallef, 2015; Park, et al. 2018). However, there is very little knowledge that is available about this with respect to the novel COVID-19 pandemic. Therefore, the current pilot study is aimed at creating a starting point for bridging the existing knowledge gaps. The current pilot study would also serve as among the first sources of knowledge for other researchers with similar interests to that of ours, given the importance of sharing lessons learnt from pilot studies (Teijlingen \& Hundley, 2001; Thabane, et al. 2019).

\section{LITERATURE REVIEW}

Some of the lived experiences and the psychosocial impacts of infectious disease outbreaks have been highlighted by researchers. For instance, Maunder et al. (2003) found negative lived experiences, stigmatisation, anxiety and stress as the common complaints presented by patients with severe acute respiratory syndrome coronavirus (SARS-COV). This was further shared in Gardener and Moallef's (2015) review which established that stigmatisation, psychotic presentations, fear of survival and infecting others, PTSD and reduced quality of life among other symptoms were apparent across all stages of postSARS.

Furthermore, literature has also demonstrated that during infectious disease outbreaks, those particularly vulnerable to mental health problems were shown to be the front-line health workers treating patients affected by the SARS-COV outbreak (Chen, et al. 2005; Gardener \& Moallef, 2015). Nickell et al. (2004) further supported this argument in their findings which established that half of the nurses working with patients of SARS-COV displayed emotional distress during the SARSCOV outbreak. Quite differently, it has been argued that both healthcare workers and the general public were shown to constitute more than one fifth of the total number of persons who experienced mental issues resulting from the SARS-COV outbreak (Sim \& Chua, 2004; Park et al. 2018). In a similar argument, Chua et al. (2004) reported that stress, fatigue and worries were among the major mental health impacts experienced by healthcare workers and the general public during SARS outbreak. Overall, this is suggestive that there is a need to consider the lived experiences and protection of the general public and specific vulnerable groups in the research and policies surrounding infectious disease outbreaks.

Infectious disease outbreaks have also been shown to require preventive and control measures which often cause changes in our lifestyles and environments (Taylor, Agho, Stevens, \& 
Raphael, 2008). These changes are often more impactful to our mental health than they are to our physical health (Wu et al. 2008). Quarantine and self-isolation are common control measures that are implemented during infectious disease outbreaks. Further, increased hygiene precaution (i.e. increased hand washing, wearing face masks, cleaning surfaces etc.) is another common control measure taken during infectious disease outbreak. Given the impacts of these control measures that are adopted to protect the general public's physical health from infectious diseases, consideration of the implications of these measures on the mental health of public is paramount (Taylor et al. 2008).

Research has further indicated negative outcomes in relation to the measures implemented during infectious disease outbreaks. For instance, Bai, et al. (2004) determined the presence of acute stress disorders in persons who experienced quarantine during the SARS-COV outbreak. It was further determined that, such persons were also likely to report detachment from others, irritability, decreased work performance, and consideration of resignation. Sprang and Silman, (2013) embarked on a comparative study between parents and children quarantined, and those not. They found that anxiety, depression, and PTSD scores were higher in children who experienced quarantine than in those who did not experience quarantine. In that same study, over a quarter of quarantined parents met the classification for PTSD compared to six percent of those not quarantined. The longitudinal impact of having experienced restricted movement during infectious disease outbreak was highlighted in Wu, et al. (2008) study which demonstrated that three years after the SARS outbreak, alcohol dependency was positively correlated with having been quarantined and selfisolated. Overall, this demonstrates that peoples' experiences of the preventive and control measures implemented during infectious disease outbreaks plays a crucial role on their mental wellbeing. It is therefore important to explore people's experiences of both the novel COVID-19, and its control/ preventive measures.

Previous literature has further shown that apart from the impact of peoples' experiences of control measures during infectious disease outbreaks, the awareness of and preparation for infectious disease outbreaks triggers mental health disturbances of anxiety (Lau et al. 2005; Lau et al. 2010). In some other studies, the levels of concern and knowledge of infectious disease outbreaks have also been identified to have impacts on mental health during such outbreaks (Almutairi et al, 2015), and this makes knowledge measurement of infectious disease an important concern during times of disease outbreaks. In another study, Taylor, et al. (2008) ascertained that greater anxiety levels were common quite early in the general public during infectious disease outbreaks, given the high levels of concern from the general public about the outbreak. The greater anxiety levels was further found to worsen by increased social distancing during infectious disease outbreaks. Therefore, this also supports our argument that the outbreak of infectious diseases have negative effects on the mental health of people. This underscores the urgency for studying the lived experiences, and mental health impacts from the novel COVID-19 pandemic (Xiang, et al. 2020).

Finally, some recent studies have been conducted on the mental health impacts from the novel COVID-19, and lived experiences of people in affected countries (Sun, et al. 2020; Nicomedes, et al. 2020; Zhang, \& Ma, 2020). However, those studies failed to consider multinational data from people who are directly or indirectly affected by the pandemic. Consequently, such studies cannot provide multinational information from a pilot study or a main study that is required to offer intervention and protection programmes for everyone affected by the novel COVID-19.

\section{Theoretical Underpinning}

Above related studies suggests a strong agreement that the outbreaks of infectious disease in our environments triggers high rates of mental issues which often include depression, and anxiety; therefore the current study would focus on depression and anxiety as the predicted mental health impacts from the COVID-19 pandemic. This is likely a consensus with the behaviorist theory of mental illness, which postulates that mental health issues such as depression and anxiety is the outcome of people's interaction with their environment (McLeod, 2015), for example people's interaction with their environments during outbreaks of infectious diseases $(\mathrm{Wu}$, et al. 2008; Sprang \& Silman, 2013). Further, in related studies, peoples' concern, knowledge, and experiences during outbreaks of infectious disease was also found to have effects on their mental health during such outbreaks (Lau et al. 2005; Lau et al. 2010; Almutairi et al, 2015), and this is a theoretical collaboration with cognitive theory of anxiety and depression (Beck, 1967), which shows that cognitive factors play key roles in predicting mental health.

\section{Research Questions}

Informed by the thorough literature review in this paper, there is currently no present multinational data from a pilot study nor a main study on the lived experiences and mental health impacts from the latest COVID-19 pandemic which is currently 
affecting 212 countries. Therefore, we presented the following research questions that were answered through a pilot study: (a). What is the feasibility of our research instrument by studying the levels of anxiety and depression in a multinational sample affected by the COVID-19 pandemic? (b). What is the feasibility of our research instrument by studying the lived experiences reported in a multinational sample affected by the COVID-19 pandemic?. Further, given the findings from Almutairi et al, (2015), and the fact that we are conducting a pilot study, we presented a third research question: (c). What is the feasibility of our research instrument by measuring the extent of knowledge of the COVID-19 pandemic in a multinational sample affected by the pandemic?. Pilot studies are often conducted to identify barriers to the recruitment of respondents in the main study (Fink, 2003). Therefore, it was asked that: (d). What barriers are likely to limit recruitment process in our main study?

\section{METHODS}

\section{Design}

This was a mixed methods study carried out to study part of a later study. The mixed method research design was adopted as it is the best design to answer our research questions that require quantitative and qualitative data (Wisdom \& Creswell, 2013). Given our study focus, and as informed by literature on how to design a mixed methods design for our kind of pilot study (Creswell \& Hirose, 2019), our study was designed to collect quantitative and qualitative data simultaneously. Therefore, our main study would be considered feasible with the mixed methods design if both the quantitative and qualitative data sources of this pilot study are able to supply sufficient information from respondents that simultaneously addresses our research questions. It would also be feasible if there is a consensus in the findings from our quantitative and qualitative studies (Wisdom \& Creswell, 2013).

\section{Sampling and Respondents}

For this pilot study, every likely respondent had equal chances of being recruited and those who responded to our survey were encouraged to share the survey with other likely respondents. Thus, we deployed a random, and snowball technique for recruiting respondents via the social media platform (Linkedin) for a very short timeframe. Our later study would, therefore, be rated as feasible if these sampling techniques and electronic survey medium enable us to recruit respondents with diverse demographics who are directly or indirectly affected by the COVID-19 pandemic. For instance, successfully recruiting respondents from more than half the total number of countries that respondents were recruited from, whose total number is more than half of the total respondents from the country with the highest number of respondents, in the short timeframe that the recruitment strategy was deployed (Lin, Lin, Yen, \& Wu, 2010).

Our study required that willing respondents could communicate (written and spoken) using English language, were 18 years and above and were directly or indirectly affected by the COVID-19 pandemic. On the other hand, our study excluded likely respondents who were not willing, who could not communicate using English language, who were less than 18 years and who were not directly or indirectly affected by the pandemic. Although our study was designed as a pilot study of everyone directly or indirectly affected by the pandemic, we excluded likely respondents who could not communicate using English language and respondents below 18 years of age, and we have addressed this in the "Strengths and limitations of our pilot study" section of this paper.

We were restricted from accessing patients with COVID-19 because of the nature of the disease, and the treatment they were receiving which limited their access to being selected for our pilot study (Adhikari et al. 2020). Therefore, our respondents (for quantitative and qualitative studies) comprised 91 members of the general population who were directly or indirectly affected by the pandemic. Their mean age was $37 \pm 9.61$ (age range 18-58 years). Further, descriptive statistics showed that, our respondents were from 14 countries (See Table 1 for further details).

\section{Instruments}

Quantitative data were collected using structured questionnaires. The Generalised Anxiety Disorder-7 (GAD7) by Spitzer, Kroenke, Williams, and Lowe (2006) was used to assess the anxiety levels of respondents. The scores on this 7 -item scale ranged from 0 to 21 and the interpretation of scores is as follows: $0-5=$ mild; $6-10=$ moderate; $11-15=$ moderately severe anxiety and $16-21=$ severe anxiety. The second questionnaire was the Patient Health Questionnaire 9 (PHQ9) by Kroenke, Spiltzer, and Williams (1999). It is a 9-item scale used to screen for the likely presence of depression in respondents. The maximum possible score on the PHQ-9 was 27 , whereas the minimum possible score was 0 . Respondents scores were interpreted as: $0-4=$ none-minimal; $5-9=$ mild; $10-14=$ moderate; $15-19=$ moderately severe and $20-27=$ 
severe. There was a supplementary respondent-rated singleitem scale, which asked respondents to rate how difficult a problem made it for them to function normally in their daily lives. The authors added this single-item scale to complement the scores of respondents in the PHQ. Scores on this singleitem scale ranged from 0 to 3 . All scales are a 4-point Likerttype scale.

The clinical instruments (GAD-7 and PHQ-9) are valid for establishing provisional diagnoses for selected DSM-IV disorders during pandemic exposures (Kroenke, Spiltzer, Williams, \& Lowe, 2010). Our current study structured these questionnaires to screen for the likely presence of anxiety and depression due to the impact of the current COVID-19 pandemic. These scales have been found to demonstrate very strong psychometric properties in various multinational samples (Cholera et al. 2014; Chibanda et al. 2016; American Psychological Association, 2019; Maroufizadeh et al. 2019). Therefore, this made these instruments a good fit for our study that was designed to target a multinational sample.

Qualitative data were collected using open-ended questions that were designed to gather data from respondents regarding their knowledge and lived experiences of the COVID-19 pandemic. Before conducting our pilot study, all research instruments received content validation from a multinational sample comprising 15 experts ( 7 clinical psychologists, 4 public health experts and 4 psychiatrists) from nine countries (Malaysia, Nigeria, the UK, China, Kenya, the Netherlands, Singapore, France, and the USA). These 15 experts were randomly selected from Twitter for this purpose, and they were not among the respondents in our pilot study, in order to avoid information contamination. We maintained close contacts with the 15 experts before and throughout our pilot study, and we informed them that they would not be included in our pilot study. All 15 experts were found to adequately comply with our instructions.

We embarked on content validation to ensure that our instruments were in line with our research objectives and questions. An agreement was reached by the 15 experts that our instruments were consistent with our research questions and objectives. An evidence for the content validation of instruments was offered by Krippendorff (2013), who established that expert judgement was imperative for ascertaining instrument appropriateness in quantitative or qualitative research. Given that the current study is a pilot phase, a high psychometric report from the research instruments in the pilot phase is suggestive of a feasibility for the full-scale study (Saunders, et al. 2009; Fink, 2003).

\section{Procedure}

A proposal that described the research purpose and procedure was submitted to the Oyo State Ministry of Health, Nigeria for the purpose of obtaining an ethical approval for this study. When designing our online survey, the first page was designed to obtain consent from the respondents after explaining the research purpose and ethical considerations and requesting the respondents to click yes if they agreed to participate in the study or click no and close the page if they declined to participate in the study.

Our quantitative and qualitative study instruments were available in only English language, and merged into a single online survey, and the link to this survey was shared by all authors to the social media platform. All authors shared the online survey through their individual social media accounts in order to increase the likelihood of recruiting respondents with diverse demographics. The online survey procedure was most suitable for collecting data from a multinational sample; it was cost saving and also suitable for the time our study was conducted (Saunders et al. 2009). This was given the total lockdown that was implemented in most parts of the world, which restricted movements of individuals. In our online survey, respondents were made to know that their participation in the study was highly voluntary and they could choose not to participate at any point of the study. They were also made to understand that there were no direct benefits for them if they participated in the study; however, an indirect benefit was stated that respondents will gain knowledge about our major findings on the social media platforms that they were selected from. Confidentiality was also maintained throughout.

The chances of self-report bias were minimised by ensuring that no personally identifiable information of respondents was requested from them. Further, we structured our online survey to restrict access to respondents who had previously completed it; therefore, it was less likely that a respondent completed our survey more than once. Also, given that the study investigated mental health impacts from the pandemic, we designed the last page of the online survey to include our contact details and we requested that respondents could contact us for e-counselling if they were down from the impacts of the pandemic. This was also supportive of the fact that the second author is a registered and experienced clinical health psychologist and the first and third authors have experienced years of practice in responding to the mental health needs of vulnerable populations. We did this as part of the protection of respondents from the likely negative impacts of the pandemic, and it was in line with 
literature's suggestion for ethical considerations during this kind of study (Adejumo \& Olorunesan, 2018).

A total of 106 respondents attempted our survey, however 91 respondents completed the survey. This gave us a completion rate of $86 \%$. After data collection, we exported data for cleaning and analyses. Quantitative data were descriptively analyzed using SPSS (v. 22.0). In related studies where our quantitative instruments were utilized on general populations, attention was given to respondents who scored a total score of 10 or above when presenting the results (Lowe, et al. 2008; Kroenke, et al. 2009). Therefore, in consonance with these related studies, we adopted the same focus when reporting respondents score on the GAD-7 and PHQ-9. On the other hand, our qualitative data were subjected to thematic analysis. Thematic analysis was most appropriate for our qualitative analysis, given that the focus of our qualitative study was aimed at understanding the patterns of lived experiences reported by respondents (Nowell, et al. 2017; Roberts, et al. 2019). Finally, although all 91 respondents had their responses reported in our quantitative results, not all had their responses reported in our final qualitative results because of the coincidence of the same responses from different respondents (Baillou, 2008).

\section{RESULTS}

Our respondents comprised 33 males and 58 females. Further, 13 of our total respondents reported having high school education as their highest education, whereas 78 reported having obtained degrees from tertiary institutions. In terms of religion, 41 respondents were Christians, 9 belonged to Islam and 41 had either no religion or other religion. In terms of the employment status, 39 were unemployed and 52 were employed. Lastly, the distribution of respondents by country showed that, of our total respondents, 11 were from China, 3 from Finland, 10 from France, 1 from Germany, 1 from Kenya, 4 from Malaysia, 3 from the Netherlands, 11 from Nigeria, 6 from Norway, 3 from Pakistan, 10 from Singapore, 7 from the United Arab Emirates, 11 from the UK and 10 were from the USA.

In Table 2, both scales were established to report very strong reliability coefficients as determined by Cronbach's alpha. Furthermore, the mean score on each scale suggests a moderate and mild level of anxiety and depression, respectively.

Table 3 shows that among all respondents, the minimum score on the GAD-7 was 0 , whereas the maximum score was 21. Further, 68 respondents had a total score $<10$, whereas 23 respondents had a total score of 10 or $>$.
Table 1. Demographic profile of respondents

\begin{tabular}{|c|c|c|}
\hline & $\mathbf{n}$ & $\%$ \\
\hline \multicolumn{3}{|l|}{ Gender } \\
\hline Male & 33 & 36.3 \\
\hline Female & 58 & 63.7 \\
\hline \multicolumn{3}{|l|}{ Highest education attained } \\
\hline High school & 13 & 14.3 \\
\hline Tertiary institution & 78 & 85.7 \\
\hline \multicolumn{3}{|l|}{ Religion } \\
\hline Christianity & 41 & 45.1 \\
\hline Islam & 9 & 9.9 \\
\hline Other religion/no religion & 41 & 45.1 \\
\hline \multicolumn{3}{|l|}{ Employment status } \\
\hline Unemployed & 39 & 42.9 \\
\hline Employed & 52 & 57.1 \\
\hline \multicolumn{3}{|l|}{ Country } \\
\hline China & 11 & 12.1 \\
\hline Finland & 3 & 3.3 \\
\hline France & 10 & 11.0 \\
\hline Germany & 1 & 1.1 \\
\hline Kenya & 1 & 1.1 \\
\hline Malaysia & 4 & 4.4 \\
\hline The Netherlands & 3 & 3.3 \\
\hline Nigeria & 11 & 12.1 \\
\hline Norway & 6 & 6.6 \\
\hline Pakistan & 3 & 3.3 \\
\hline Singapore & 10 & 11.0 \\
\hline United Arab Emirates & 7 & 7.7 \\
\hline UK & 11 & 12.1 \\
\hline USA & 10 & 11.0 \\
\hline
\end{tabular}

The results from the supplementary single-item scale showed that, 33 respondents reported no difficulty in their daily living as a result of the COVID-19 pandemic. Further, 43 respondents reported they found it somewhat difficult, whereas 12 respondents reported that it was very difficult for them in terms of their daily living due to the COVID-19 pandemic. Lastly, three respondents reported extreme difficulty in terms of their daily living due to the pandemic.

Table 5 shows that among all respondents, the minimum score on the PHQ-9 scale was 0, whereas the maximum score was 25 . Further, 70 respondents had a total score $<10$, whereas 21 respondents had a total score of 10 or $>$. 
Table 2. Descriptive statistics and Cronbach's alpha scale

\begin{tabular}{|c|c|c|c|}
\hline Scale & Mean & SD & a \\
\hline GAD-7 & 6 & 5.54 & 0.90 \\
\hline PHQ-9 & 6.4 & 5.84 & 0.88 \\
\hline
\end{tabular}

GAD-7, Generalised Anxiety Disorder-7; PHQ-9, Patient Health Questionnaire 9

Table 3. Distribution of respondents by total score on the GAD-7 scale

\begin{tabular}{|c|c|c|}
\hline Total score & Freq. & $\%$ \\
\hline 0 & 14 & 15.4 \\
\hline 1 & 9 & 9.9 \\
\hline 2 & 10 & 11.0 \\
\hline 3 & 10 & 11.0 \\
\hline 4 & 8 & 8.8 \\
\hline 5 & 4 & 4.4 \\
\hline 6 & 5 & 5.5 \\
\hline 7 & 1 & 1.1 \\
\hline 8 & 5 & 5.5 \\
\hline 9 & 2 & 2.2 \\
\hline 10 & 4 & 4.4 \\
\hline 11 & 3 & 3.3 \\
\hline 12 & 4 & 4.4 \\
\hline 13 & 3 & 3.3 \\
\hline 15 & 3 & 3.3 \\
\hline 16 & 2 & 2.2 \\
\hline 20 & 2 & 2.2 \\
\hline 21 & 2 & 2.2 \\
\hline
\end{tabular}

Table 4. Distribution of respondents by total score on the supplementary single-item scale

\begin{tabular}{|c|c|c|}
\hline Total score & Freq. & $\%$ \\
\hline 0 & 33 & 36.3 \\
\hline 1 & 43 & 47.3 \\
\hline 2 & 12 & 13.2 \\
\hline 3 & 3 & 3.3 \\
\hline
\end{tabular}

The results shown in Table 6 reveal that more than half of the respondents had high knowledge level of COVID-19. The criteria for judging their knowledge included: history of the disease, the causal agent, early and late symptoms, the transmission process, preventive measures, the most vulnerable population and help-seeking approaches for persons living with or at risk of COVID-19. Therefore, respondents who met
Table 5. Distribution of respondents by total score on the PHQ-9 scale

\begin{tabular}{|c|c|c|}
\hline Total score & Freq. & $\%$ \\
\hline 0 & 14 & 15.4 \\
\hline 1 & 5 & 5.5 \\
\hline 2 & 10 & 11.0 \\
\hline 3 & 5 & 5.5 \\
\hline 4 & 9 & 9.9 \\
\hline 5 & 7 & 7.7 \\
\hline 6 & 3 & 3.3 \\
\hline 7 & 8 & 8.8 \\
\hline 8 & 4 & 4.4 \\
\hline 9 & 5 & 5.5 \\
\hline 10 & 4 & 4.4 \\
\hline 12 & 4 & 4.4 \\
\hline 13 & 3 & 3.3 \\
\hline 14 & 1 & 1.1 \\
\hline 15 & 1 & 1.1 \\
\hline 18 & 2 & 2.2 \\
\hline 19 & 3 & 3.3 \\
\hline 21 & 1 & 1.1 \\
\hline 22 & 1 & 1.1 \\
\hline 25 & 1 & 1.1 \\
\hline
\end{tabular}

PHQ-9, Patient Health Questionnaire 9

Table 6. Summary of respondents' knowledge of COVID-19 What do you know about COVID-19? Please, freely expand your responses.

\begin{tabular}{|c|c|c|}
\hline $\begin{array}{c}\text { High } \\
\text { knowledge }\end{array}$ & $\begin{array}{c}\text { Partial } \\
\text { knowledge }\end{array}$ & $\begin{array}{c}\text { Poor knowledge/no } \\
\text { knowledge }\end{array}$ \\
\hline $58(63.7 \%)$ & $30(33.0 \%)$ & $3(3.3 \%)$ \\
\hline
\end{tabular}

all the criteria were grouped as having high knowledge, while those who met some but not all these criteria were grouped as having partial knowledge. On the other hand, respondents who did not meet any of these criteria were grouped as having poor knowledge or no knowledge.

We noticed that respondents who demonstrated partial knowledge often reported good knowledge on either the history of the disease, early and late symptoms, or transmission process of the disease. Furthermore, regarding the total respondents who had high knowledge level of COVID-19, they mostly stated that the major sources from which they gained knowledge were 
from the television, radio, online academic papers and other internet materials.

\section{Qualitative Results}

This section presents the qualitative findings which demonstrated the lived experiences of respondents from the COVID-19 pandemic. Responses were labelled with the gender, age and country of respondent. The following themes emerged.

\section{Changes in lifestyle}

This was the first theme that emerged, showing that most respondents reported changes in their lifestyle due to the pandemic. These are seen in the following responses:

People now have only one walk a day, otherwise stay at home...I'm unable to see close family. We have to use facemask if going out, and there is not enough facemask in this country. We have to use hand gel [hand sanitizer] after opening any post. Social distancing is another. We are unable to get food delivered, and me and mum can't go out as we are immunocompromised. (Female 35 years, UK)

... Because of the pandemic, I rarely leave the house and have only met around 3 friends (outside and with 2 meter distance) during the last 6 weeks. (Female 26 years, Germany)

My experiences are confinement, working from home, and holiday cancellation.... I moved to take care of my elderly parents, and I am far away from my partner. I am also stressed about the health status of family and friends. We have been doing lots of e-communication than usual. (Female 58 years, France)

I now socialize with friends via more than one social media that facilitate video chats... I value my one walk a day routine as precious and I never miss it. But I cannot do this routine now.... (Female 37 years, Singapore)

Lifestyles are compulsorily changing now, people are forced to adapt in such a short time.... (Male 24 years, Singapore)

Similarly, a set of respondents opined the following:

I and members of my family have had to change our routines drastically... I live apart from my family, and I work as normal since I am one of the frontline staff in a hospital, looking after Covid-19 positive patients. When I'm not working I don't like to go out at all, in case I have become infected at work. I only go shopping once per week at times designated for NHS staff only. (Female 23 years, UK)

It has caused a massive disorientation. Life as we know has now changed. (Female 20 years, Pakistan)

... I traveled, and before I could go home, I was quarantined for 2 weeks, and I was not allowed out of my room - this is not what I am used to.... (Male 18 years, China)

... To go grocery shopping, you have to queue outside and keep at least $2 \mathrm{~m}$ distance from everyone around you. In my house I was quarantined for 2 weeks with my both parents. Although we haven't been tested, I'm certain we all have COVID-19 as my dad contracted it from his office as there was a confirmed case there.... (Female 18 years, USA)

The pandemic has a lot of impacts such like my dad that lives in another country can't travel to visit us in the UK, weddings being cancelled, and lives being lost. Also, churches being closed down and, I am not able to live normally. It's all sad! (Female 30 years, UK)

This has caused limited mobility outside the house, and we have to go through tedious process of wearing face masks and gloves.... (Male 28 years, Kenya)

Another set of respondents similarly stated as follows:

... I have not been able to see my parents, and we now leave the house to get food and go for a walk only once a day. Also, I cannot see any of my friends face-to-face. (Female 26 years, Singapore).

I have felt little change to my life except in my study routine.... Many of my friends are going crazy about the changes of being trapped at home. (Male 29 years, China)

Since the pandemic, I haven't been able to go out and meet up with friends.... Foods have become less varied and more mundane.... Many people are unmotivated (Male 20 years, Pakistan)

I live in the UK, but home is in Ireland. This is a short journey (40 min flight or 4 hour boat) but I cannot travel home to see my family, which I do once a month ... being apart from my family in such a troublesome time, is the biggest impact for me. (Female 24 years, UK) 
Covid-19 has affected first the rest of my family before it has affected me as they are all in Spain so they began their isolation about 10 days before me, and it means they have to change their lifestyle by having to always stay at home.... (Female 23 years, USA)

Everything has changed... I am unable to go outside to see people.... (Female 42 years, China)

We've had to stay at home due to the pandemic, so the whole family is home, which isn't usual. (Female 26 years, Nigeria)

\section{Disruption of Schooling activities}

Some other respondents reported disruption of their schooling activities:

... My university in the UK was closed as well as bars and pubs, so all my classes stopped and are being done online. The pandemic also made a lot of friends to leave the university campus, and go back home with their families... I feel isolated in the UK. (Female 22 years, UK)

It impacted the daily routine of my family as now most offices and schools have stopped operations. (Female 54 years, Malaysia)

I have school from home now and I cannot go outside, my school exams have also been cancelled.... (Female 18 years, Nigeria)

Due to the virus, I have had to work from home and limit all travel to essential places. This also caused cancelation of university lectures and changes to how I'll be examined this year.... (Male 34 years, France)

... I am confined in my apartment. My academics are suffering from it.... (Male 23 years, France)

Another respondent reported changes in schooling activities and difficulty coping with these changes. Furthermore, he differently reported increased fighting among people staying at home due to the lockdown:

The pandemic caused schools to close, and this has made teaching harder, as more planning is required to teach online. Also, the ongoing lockdown which has kept people indoors has also increased fighting indoor and boredom (Male 51 years, China)

\section{Impact on Health Service Options}

A 35-year-old respondent from Norway stated that the pandemic has had impacts on the options to get health services for illnesses other than COVID-19:

The virus in itself has not had any impact, however the measures taken in response to it has impacted the meaning of life, and the options to get health services for other cases than COVID-19. (Male 35 years, Norway)

\section{Disruption of Works and Economy}

Another set of respondents stated that the COVID-19 pandemic has disrupted their works and country's economy:

COVID-19 has brought some changes to work procedures for me, and some people I know lost their jobs (different companies), including my sister. (Female 40 years, USA)

... Because of this, the company my dad works for is furloughing people. Businesses generally are struggling, we will most likely be in a recession when Covid-19 is over. (Female 22 years, China)

... Personally, it has affected job security as my salary has been reduced by 20\% and shifts increased. (Female 24 years, Nigeria)

The pandemic has introduced gradual increase of food prices. Restricted movements via curfews, flights restriction, and implementation of distant teaching. (Female 44 years, UAE)

... I have friends who lost their jobs due to lack of work as businesses are closed, and the companies cannot afford to pay them due to the pandemic.... (Female 22 years, Singapore)

Similarly, some respondents reported thus:

It has caused a major slowdown in activities on an international scale. People are now being forced to stay at home, the prices of items especially consumables are up. (Female 23 years, Nigeria)

I'm a small business owner so this has disrupted my source of income.... (Male 27 years, UAE)

Due to this, I have lost a potential job (a university suspended hiring), my partner's work hours have been reduced and he is 
working from home and we are all communicating via online platforms and phone calls. (Female 38 years, USA)

I am a teacher so I am having to work from home, and this is providing challenges on how to educate children from home, especially as I am a year one teacher so the children cannot do any online independent work. My partner's work has also been put on furlough as not many companies are recruiting, and therefore his business is struggling because they have no work.... (Female 23 years, UK)

For me, it has led to the shutdown of my laboratory and this means I've had to stop all research activities. This also means I've been at home nearly all day every day, which is much of a slower pace than I'm used to. (Male 24 years, UK)

Due to the pandemic, I have been working from home for about a month, and the discipline to keep working is hard (Male 36 years, UAE)

We have been telecommuting for work for about a month.... Local restaurants and many stores are closed.... Initially some essentials were in short supply at stores (e.g., toilet paper, bread) but supply has mostly recovered. (Female 32 years, USA)

Also, this respondent stated same; in addition, he stated that the pandemic caused an end to his dating relationship in Spain:

I was working and living in Spain as an Au Pair/English Teacher. I had to come home in the end as my classes were cancelled. This was a difficult decision as I was also in a relationship out there. I decided to end the relationship too. Now I am in lockdown and looking for future opportunities. (Male 24 years, Singapore)

Another 53-year-old UK respondent stated thus:

This pandemic has brought about lockdown, and massive unemployment. Many local businesses are going bust. This is likely to have a catastrophic effect on the economy which will kill more people than the virus itself ever could (Male 53 years, UK)

\section{Anxious and Worrisome Impacts}

The respondents stated that their major lived experience from the COVID-19 pandemic was anxiety:

I feel more anxious, irritated and frustrated, isolated, lonely and all in all more and more miserable and tired of this whole pandemic situation. (Female 31 years, Finland)
... I am worried about my health due to existing conditions and worried about my parents due to their age and health. (Female 22 years, Norway)

Because of the pandemic, I had to cancel my travelling plans and return from the US to Malaysia sooner than planned. Malaysia implemented the Movement Control Order (MCO) around the same time I returned.... That put my family and me at enormous stress as the fear of the inability to get home caught up with us.... (Male 24 years, Malaysia)

Another respondent simply emphasised:

Confusion and fear!!! [Emphasises] (Male 37 years, Finland)

Another respondent reported that his major anxious concern was centred on the fear of contracting the virus:

... Fear of catching the virus, which has led to an increase in mental health issues for me and others. (Male 26 years, Nigeria)

A respondent from the Netherlands stated that the psychological impacts from the worries of the pandemic were wearing him down:

... It has a subtle psychological impact that is slowly wearing me down. I seem to lack concentration in almost anything I try to do. I feel anxious whilst time flows around me. (Male 23 years, the Netherlands).

A UAE respondent whose dad was vulnerable stated that he and his friends were very worried about the spread and impact of COVID-19:

I have had to adapt to learning at home but I am still worried. My dad has been classified as a vulnerable person and has to stay inside for 4 months; meanwhile my mum is a key worker. Half of my best friends are key workers and are also worried with the spread and impact in our hometown. (Male 24 years, UAE)

A Nigerian female respondent stated that she was scared of the pandemic, and she likely developed obsessive compulsive disorder (OCD) which she thought would have been more harmful than the COVID-19 pandemic itself:

Definitely fear, I was very scared when I heard about it although my husband wasn't moved and still isn't as he believes there are deadlier diseases, and COVID-19 isn't really 
one of them. But I was scared, and I started becoming extra careful. After a while, I stopped being scared because I realized that fear and exhibiting OCD symptoms might kill faster than the virus itself. (Female 23, Nigeria)

A UK respondent who was recovering from anxiety before the onset of the pandemic stated that the pandemic was having impacts on her recovery process:

We have been staying inside as much as possible which is having an impact on my mental health. As someone who is recovering from anxiety (and recovering well before the pandemic), I can feel my mental health starting to turn and I am having to work over drive to stay on my path of recovery. It is difficult generally having your whole life changed in an instant. (Female 33, UK)

Another respondent from China stated that the pandemic had mental impacts typical of fears and worries:

It has had a mental (fear and worries), emotional and financial impact on myself, and a similar impact on those around me. (Female 18 years, China)

Similarly, the respondents stated that:

It has disrupted daily activities, has increased fear, and anxiety. (Male 27 years, Nigeria).

Restricted movement, anxiety, suspicion and fear. (Female 18 years, Norway)

... Because of the pandemic, I have a fear factor on family members with underlying conditions. (Male 37 years, France)

... Some of my friends are vulnerable to COVID-19 and that worries me as I would hate them to get ill. (Female 35 years, Malaysia)

... It has made me feel anxious about leaving the house. I'm scared I'll catch the virus and die. I'm scared my family and friends will die. My friends and family are very anxious about it also. It's all they talk about now. (Female 22 years, UAE)

\section{DISCUSSION}

Our pilot study found that more respondents had scores of 10 or greater on the GAD-7, as compared with the PHQ-9. We also found that more than half $(58,63.7 \%)$ of the total respondents demonstrated high knowledge level of the COVID-19 pandemic, with their major information sources as television, radio, online academic papers, and other internet information. These findings were obtained from our quantitative insight, and they were in consonance with literature where anxiety and depressive symptoms have been reported among people who were directly or indirectly affected by infectious disease outbreaks (Lau, et al. 2005; Maunder, et al. 2003; Taylor et al. 2008; Sprang \& Silman, 2013; Gardener \& Moallef, 2015). Going further, the findings from our knowledge assessment of COVID-19 was in agreement with Lau, et al. (2005) who found in a diverse sample that although respondents were not psychologically prepared for the SARS-COV outbreak, more than half of the total respondents showed good knowledge of the disease, and this good knowledge possibly limited the mental health impacts of the disease outbreak on respondents. Therefore, the fact that more than half of the total respondents in our pilot study showed high knowledge level of the COVID-19 pandemic could likely justify the extent of mental health impacts (anxiety \& depression) resulting from the COVID-19 pandemic that was observed in our results.

Our qualitative study explored the lived experiences of respondents from the COVID-19 pandemic, and the findings revealed five (5) themes which were; changes in lifestyles which was the first theme reported by most respondents in the qualitative study. Second theme was the disruption of schooling activities. Third was impact on options for health services. Fourth was disruption of works and economy, and fifth was anxious and worrisome impacts of the COVID-19 pandemic. In further details, the first theme demonstrated that most respondents in our qualitative study experienced changes in their lifestyles. For instance, restricted movements, increased socializing via social media due to lockdowns, and increased use of face masks and hand sanitizers. Further, it was obvious that most respondents were not used to nor prepared for these changes in lifestyle before the pandemic started. Therefore, the first theme which accommodated most responses from our respondents was in consonance with literature which submitted that preventive/control measures implemented during infectious diseases outbreaks play crucial roles in affecting people (Bai, et al. 2004; Sprang \& Silman, 2013).

The second theme which showed that respondents felt disruption of schooling activities as their major lived experience showed that most respondents in this theme stated that their school had either stopped operation or they had to do schooling from home which they were obviously not used to before COVID-19 became a pandemic. Also, some respondents in this theme reported that their examination has been cancelled due to the pandemic. The third theme was 
formed from a respondent who reported that the COVID-19 pandemic has affected his health service options for illnesses other than COVID-19. This is, therefore, suggestive that clinical populations other than patients with COVID-19 may have limited practice of professional help seeking for any health problem that they are living with, and this is due to the novel COVID-19 which has disrupted the normal way of life for almost everyone.

The fourth theme showed that the major experience of some respondents caused by the pandemic was the disruption of works and economy caused by the pandemic. In further detail, respondents in this theme stated that the COVID-19 pandemic had significantly affected their work procedures, and they were not prepared for this. Some others stated that the pandemic had affected their job security, caused their businesses to struggle and increased telecommuting for them. There were also respondents who strongly stated that there was a drastic increase in the prices of foods and other items due to the pandemic. Some other respondents in this theme also reported that they were currently experiencing scarcity of essential items at supermarkets. Therefore, the first, second, third and fourth themes identified in our study can be linked to the fact that the novel COVID-19 pandemic has brought about drastic changes among our respondents in these themes, and it is also evident that our respondents were mostly not prepared for these changes as reflected in most of their responses in the "Qualitative results" section of this paper. From the responses of respondents in these themes, it is also evident that most of them are still on the process of coping with the changes caused by the novel pandemic. The first, second, third and fourth themes are thus quite in agreement with Lau et al. (2005), who reported that psychological preparation for infectious disease outbreak was imperative for coping during such outbreaks.

Our last theme which showed that anxious and worrisome impacts were the major experiences of some respondents demonstrated fears about contracting or dying from COVID-19. It also demonstrated fears about family members who were most vulnerable because of certain underlying illnesses that they had. It also established fears about the pandemic from conversations that respondents have been having with their friends in recent days. Given the anxious concerns of respondents, the last theme suggests that the pandemic could have triggered anxiety because of likely catastrophising which the respondents engaged in, and this is a theoretical agreement with the cognitive theory of mental illness (Beck, 1967). This was also supported by the last response in the qualitative study which was from a female, who stated that she was scared that her family and friends will contract the virus and die from it. The last theme reported in our study was in collaboration with Gardener and Moallef (2015) who argued that fear of contracting, fear of survival and fear of infecting others were among the experiences of people during infectious disease outbreaks. It was also in agreement with Maunder et al. (2003) who opined that anxiety was common among people during the outbreak of SARS-COV.

Finally, apart from the consistency of our findings with literature, we also observed that there was a consensus between our quantitative and qualitative findings (Wisdom \& Creswell, 2013). For instance, in our quantitative results, we observed that in comparison with the PHQ-9, more respondents submitted total scores of 10 or greater on the GAD-7. An implication of these scores is that the COVID-19 pandemic had more anxious than depressive impacts on our respondents. This was therefore in agreement with our qualitative results where in comparison with reporting depressive experience about the COVID-19 pandemic, respondents often reported anxious experiences about COVID-19 pandemic. This consensus is suggestive that our research instruments were valid, by truly measuring what they were designed to measure. It is also an indication that the content validation conducted before this pilot study which we reported in "the methods section" of this paper was efficacious.

\section{LESSONS LEARNT FROM OUR PILOT STUDY}

Our study being a pilot study has offered great insights into what is actually needed for our main multinational study. These insights include that the quantitative and qualitative instruments used in this pilot phase are feasible for an actual multinational study of this kind, and this is given the high psychometric reports, and consensus in findings from the data gathered by our instruments (Fink, 2003; Saunders, et al. 2009; Wisdom \& Creswell, 2013). Another insight offered suggests that the inclusion of measures that quantifies psychosocial preparedness for pandemics may be considered in the later study given that most respondents' reported lived experience was changes in lifestyle, disruption of schools, works and economy. These respondents also submitted unpreparedness for these changes, and this probably might have triggered the extent of anxiety levels found in this pilot study. The psychosocial preparedness for pandemics measure may thus be considered for the purpose of analyzing the interactions between scores on such measure and scores on the GAD-7.

Further, we dedicated a very short timeframe with our little available financial resources to the recruitment of respondents for this pilot study via Linkedin. Despite this, our recruitment strategy enabled us to recruit respondents from fourteen (14) 
countries, and the highest number of respondents from each country was 11 , with 8 countries having more than half of 11 respondents. This is a plus to our recruitment strategy (Lin, et al. 2010); it has thus revealed that, in order to access a large sample that is representative enough of everyone who are directly or indirectly affected by the pandemic, a sufficient timeframe with adequate financial resources, and more social media platforms will be required. The ability of this pilot study to reveal the requirements for our main study is in line with scholars' opinions about the importance of pilot studies (Teijlingen \& Hundley, 2001; Maunder, 2009; Thabane, et al. 2019; Malmqvist, et al. 2019; Bidarbakhtnia, 2020).

Another offered insight suggests that, it was not obvious whether patients or survivors of COVID-19 were among our respondents, as we were restricted from accessing them (Adhikari, et al. 2020). Therefore, the need to ensure that this population is included in a later study is also underscored. Given all insights gained from our pilot study, it thus presents a consensus with the criteria for judging the feasibility of our main study that was mentioned in our methods section (Fink, 2003; Saunders, et al. 2009; Lin, et al. 2010; Wisdom \& Creswell, 2013).

From all above, there is a very strong agreement that the current study succeeded in providing great insights for our main study. Therefore, the main study is very feasible, with minor addition needed on the protocol.

\section{Recommendation}

Given our findings from the knowledge assessment of COVID-19, we recommend that appropriate information sharing should be strengthened during pandemics in order to increase public awareness during pandemics. Second, we recommend that appropriate information sharing through television, radio, online academic papers, and other internet materials should be encouraged given the finding that most respondents stated these as their sources of knowledge about COVID-19. There is also the need to make these sources of knowledge accessible to others who lack access to them.

Going further, we recommend that adaptive coping skill training should be a concern for counselors, therapists and other health workers involved in works with persons affected by the COVID-19 pandemic. Such adaptive coping skill training should focus on decreasing the adverse impacts of the pandemic by training people to adapt to the changes or disruptions that are brought about by the pandemic.
Further, we recommend the need for psychosocial preparation due to the uncertainty of pandemics. In addition, averting the likely adverse economic impacts from pandemics should be of concern to governments and other stakeholders during pandemics. Finally, we recommend that governments and other stakeholders should be cautious about the likelihood of limited health service options for the general public during pandemics.

\section{Strengths and Limitations of Our Pilot Study}

Our pilot study showed high validity and reliability of our research instruments on a multinational sample, and this met the relevance of pilot studies (Saunders, et al. 2009). Another strength of our study was the very active efforts deployed by the researchers to minimize the occurrence of confounding factors. Therefore, the reported feasibility of our main study could minimally be undermined by confounding factors. Another strength of our study was the e-counseling services we offered to respondents to reduce the adverse impacts of COVID-19 pandemic on our respondents. On the other hand, our pilot study on 91 respondents offered great insights into what is required for a later study, it however failed to meet the minimum requirement of sample size for pilot studies of this kind (Fink, 2003), and this was attributed to our available resources. It is thus suggestive that a pilot study on a bigger sample size might have offered greater insights into what would be required for a later study.

Another limitation of our study is that our study excluded likely respondents who could not communicate using English language, and this was because our available financial resources could not accommodate paying for the services of an interpreter of any other language. We also excluded likely respondents who were below age 18 years, and this was because of the sensitivity of informed consent for persons below adult age. There is thus the need to consider these populations in a later study because they were excluded from our pilot study.

\section{CONCLUSION AND FURTHER RESEARCH}

We conclude that our main study is very feasible, with minor additions required on the protocol. Finally, full-scale studies are needed in order to provide sufficiently educated decisions that are aimed at addressing the mental health needs of everyone who are directly or indirectly affected by the experience of the novel COVID-19 pandemic. 


\section{ACKNOWLEDGEMENT}

We sincerely acknowledge all respondents who dedicated their time to fill our survey.

\section{CONTRIBUTION OF AUTHORS}

All four authors contributed equally towards the conceptualisation, design, data collection/analyses, article writing and proofreading of final manuscript.

\section{CONFLICT OF INTEREST}

We declare that there are no conflicts of interest for our study.

\section{ETHICS}

Our study was carried out in accordance with the ethical standards of the 1964 Helsinki Declaration. Ethical approval was received from the Ethical Review Board of Oyo state, Nigeria Ministry of Health.

\section{FUNDING}

There was no funding for this study.

\section{INFORMED CONSENT}

Consent was obtained via online consent form from all respondents in our study.

\section{REFERENCES}

Adejumo, A. 0., \& Olorunesan, A. T. Ethical issues in clinical psychology research in

Nigeria and coping techniques. British Journal of Psychology Research, 2018; 6(2): 47-62.

Adhikari, S., Meng, S., Wu, Y., et al. Epidemiology, causes, clinical manifestation, and diagnosis, prevention, and control of coronavirus disease (COVID-19) during the early outbreak period: A scoping review. Infect Dis Poverty, 2020; 9, 29.

Almutairi, K. M., Al Helih, E. M., Moussa, M., Boshaiqah, A. E., Alajilan, A. S., Vinluan, J. M., \& Almutairi, A.. Awareness, attitudes, and practices related to coronavirus pandemic among public in Saudi Arabia. Family \& Community Health. The Journal of Health Promotion \& Maintenance, 2015; 38(4), 332-340.

American Psychological Association. Patient health questionnaire (PHQ-9 \& PHQ-2). 2019; Retrieved from https://www.apa.org/pi/ about/publications/caregivers/practice-settings/assessment/ tools/patient-health.

Bai, Y., Lin, C. C., Lin, C. Y., Chue, C. M. \& Chou, P. Survey of stress reactions among healthcare workers involved with the SARS outbreak. Psychiatr Serv. 2004; 55(9): 1055-1057.

Baillou, J. Open-ended questions. In P. J. Lavrakas (Ed.), Encyclopedia of survey research methods, 2008; (pp. 548-550). Thousand Oaks, CA: SAGE.

Beck, A. T. Depression: Clinical, experimental and theoretical aspects. New York: 1967; Harper and Row.
Bidarbakhtnia, A. Survey under lockdown; A pandemic lesson. Stats Brief, April, 2020, 23: 1-7

Chen C. S., Wu H. Y., Yang P., \& Yen C. F. Psychological distress of nurses in Taiwan who worked during the outbreak of SARS. Psychiatric Services 2005; 56(1): 76-79.

Chibanda, D., Verhey, R., Gibson, L. J., et al. Validation of screening tools for depression and anxiety disorders in a primary care population with high HIV prevalence in Zimbabwe. J Affect Diord, 2016; 198: 50-55.

Cholera, R., Gaynes, B., Pence, B., et al. Validity of the patient health questionnaire- 9 to screen for depression in high-HIV burden primary healthcare clinic in Johannesburg, South Africa. J Affect Disord, 2014; 167: 160-167.

Chua, S. E., Cheung, V., et al. Stress and psychological impact on SARS patients during the outbreak. Can J. Psychiatry, 2004; 49(6): 385-390.

Creswell, J. W., \& Hirose, M. Mixed methods and survey research in family medicine and community health. Fam Med Com Health, 2019; 7: e000086.

Fink, A. How to sample in surveys. 2nd Edition. 2003; Thousand Oaks: Sage.

Gardener, P. J., \& Moallef, P. Psychological impacts on SARS survivors: Critical review of the English language literature. Canadian Psychology/Psychologie Canadienne, 2015; 56(1): 123135. 
Kadam, P., \& Bhalerao, S. Sample size calculation. Int J. Ayurveda Res. 2010; 1(1): 55-57.

Krippendorff, K. Content analysis. An introduction to its methodology (3rd ed). 2013; California, Ca: Sage Publications.

Kroenke, K., Spiltzer, R. L., \& Williams, J. W. Validity of a brief depression severity measure. J Gen Intern Med, 1999; 16(9): 606613.

Kroenke, K., Strine, T. W., Spitzer, R. L., Williams, J. B., Berry, J. T., Mokdad, A. H. The PHQ-8 as a measure of current depression in the general population. J Affect Disord. 2009; 114(1-3): 163-173.

Kroenke, K., Spiltzer, R. L., Williams, J. W., \& Lowe, B. (2010). The patient health questionnaire somatic, anxiety, and depressive symptom scales: A systematic review. Gen Hosp Psychiatry, 2010; 32(4): 345-359.

Lau J. T, Yang X, Pang E, Tsui H. Y, Wong E, \& Wing YK. (2005) SARS-related perceptions in Hong Kong. Emerging Infectious Disease, 2005; 11(3):417-424.

Lau, E. H., Hsiung, C. A., Cowling, B. J. et al. (2010) A comparative epidemiologic analysis of SARS in Hong Kong, Beijing and Taiwan. BMC Infect Dis, 2010; 10, 50.

Lazarus, P.J., Jimerson, S.R., \& Broch, S.E. Helping children after a natural disaster: Information for parents and teachers. In S. E. Brock, P.J. Lazarus, \& S. R. Jimerson (Eds.), 2003; Best practices in school crisis prevention and intervention (pp. 435-450). Bethesda, MD: National Association of School Psychologists.

Lin, C., Lin, C., Yen, D. C. et al. The integrated information architecture: A pilot study approach to leveraging logistics management with regard to influenza preparedness. J Med Syst, 2010; 36, 187-200.

Lowe, B., Decker, O., Muller, S., et al. Validation and standardization of the generalized anxiety disorder screener (GAD-7) in the general population. Med Care. 2008; 46(3): 266-274.

Malmqvist, J., et al. Conducting the pilot study: A neglected part of the research process? Methodological findings supporting the importance of piloting in qualitative research studies. International Journal of Qualitative Methods, 2019; https://doi. org/10.1177/1609406919878341.

Maroufizadeh, S., Omani-Samani, R., Almasi-Hashiani, A., et al. The reliability and validity of the patient health questionnaire- 9
(PHQ-9) and PHQ-2 in patients with fertility. Reproductive Health, 2019; 16: 137.

Maunder, R., Hunter, J., Vincent L., et al. The immediate psychological and occupational impact of the 2003 SARS outbreak in a teaching hospital. Canadian Medical Association Journal, 2003; 168 (10), 1245-1251.

Maunder, R. Was SARS a mental health catastrophe? General Hospital Psychiatry, 2009; 31(4): 316-317.

McLeod, S. Psychological theories of depression. Retrieved from 2015; https://www.simplypsychology.org/depression.html

Nickell, L. A., Crighton, E. J., Tracy, C. S., Al-Enazy, H., Bolaji, Y., Hanjrah, S., Hussain, A., Makhlouf, S., \& Upshur, R. E. Psychosocial effects of SARS on hospital staff: survey of a large tertiary care institution. CMAJ : Canadian Medical Association journal, 2004; 170(5), 793-798. https://doi.org/10.1503/cmaj.1031077

Nicomedes, C. J., Avila, R. M., \& Arpia, H. M. The lived experiences of Filipino front liners during COVID-19 outbreak. 2020; doi: 10.13140/RG.2.2.21221.35046/1

Nowell, L. S., et al. Thematic analysis: Striving to meet the trustworthiness criteria. International Journal of Qualitative Methods, 2017; https://doi.org/10.1177/1609406917733847

Park, J. S., Lee, E. H., Park, N. R., \& Choi, Y. H. (2018). Mental health of nurses working at a government-designated hospital during a MERS-COV outbreak: A cross-sectional study. Archives of Psychiatric Nursing, 2018; 32(1), 2-6.

Saunders, M. et al. Research methods for business students. 2009; Pearson, New York.

Roberts, K., Dowell, A., \& Nie, J. Attempting rigor and replicability in thematic analysis of qualitative research data: A case study of codebook development. BMC Med Res Methodol, 2019; 19, 66.

Rothan, H. A., \& Byrareddy, S. N. The epidemiology and pathogenesis of coronavirus disease (COVID-19) outbreak. Journal of Autoimmunity, 2020; 109, 102433.

Saunders, M., Lewis, P., \& Thornhill, A. Research methods for business students. Pearson, 2009; New York.

Sim, K., \& Chua, H.C. The psychological impact of SARS: A matter of heart and mind. Canadian Medical Association Journal, 2004; 170(5), 811-812. 
Spitzer, R. L., Kroenke, K., Williams, J. W., \& Lowe, B. A brief measure for assessing generalized anxiety disorder: The GAD-7. Arch Intern Med, 2006; 166: 1092-1097.

Sprang G, \& Silman M. Posttraumatic stress disorder in parents and youth after health-related disasters. Disaster Med Public Health Prep, 2013; 7: 105-110.

Sun, N, Wei, L., Shi, S., et al. A qualitative study on the psychological experience of caregivers of COVID-19 patients. American Journal of Infection Control, 2020; 48: 592-598.

Taylor, M. R., Agho, K. E., Stevens, G. J., \& Raphael, B. Factors influencing psychological distress during a disease epidemic: Data from Australia's first outbreak of equine influenza. BMC Public Health, 2008; 8, 347.

Thabane, L. et al. Population health intervention research: What is the place for pilot studies? Trials, 2019; 20, 309.

Teijlingen, E. R. \& Hundley, V. The importance of pilot studies. Social Research Update, 2001; vol. 35.

WHO Coronavirus

https://www.who.int/emergencies/diseases/novelcoronavirus-2019

https://www.who.int/health-topics/coronavirus

https://www.who.int/emergencies/diseases/novelcoronavirus-2019

Wisdom, J., \& Creswell, J. W. Mixed methods: integrating quantitative and qualitative data collection and analysis while studying patient-centered medical home models. Rockville: Agency for Healthcare Research and Quality. February 2013. AHRQ Publication No. 13-0028-EF

Wu P., Liu X., Fang Y., et al. Alcohol abuse/dependence symptoms among hospital employees exposed to a SARS outbreak. Alcohol Alcohol, 2008; 43(6): 706-712.

Xiang, Y., et al. Timely mental health care for the 2019 novel coronavirus outbreak is urgently needed. The Lancet Psychiatry; 2020, 7: 3 .

Zhang, Y., \& Ma, Z. F. Impact of the COVID-19 pandemic on mental health and quality of life among local residents in Liaoning province, China: A cross-sectional study. International Journal of Environmental Research and Public Health, 2020; 17(7): 2381. 